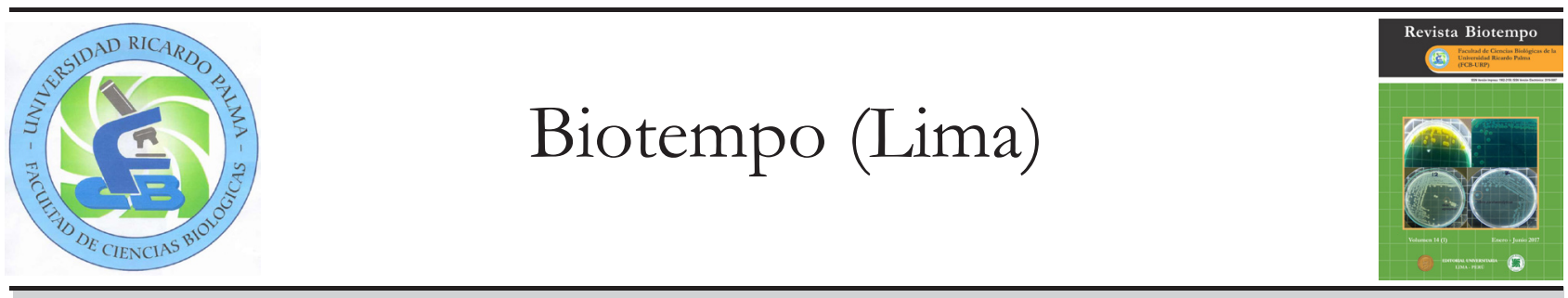

ORIGINAL ARTICLE/ ARTÍCULO ORIGINAL

LEPTOSPIROSIS AND ITS RELATIONSHIP WITH SOME CLIMATIC VARIABLES IN THE MUNICIPALITY OF SANTA CLARA, VILLA CLARA, CUBA

\title{
LEPTOSPIROSIS Y SU RELACIÓN CON ALGUNAS VARIABLES CLIMÁTICAS EN EL MUNICIPIO DE SANTA CLARA, VILLA CLARA, CUBA
}

Jorge Abilio Pérez Bastida ${ }^{1 *}$; Ricardo Osés Rodríguez ${ }^{2}$; Rigoberto Fimia Duarte ${ }^{1}$; Omelio Cepero Rodríguez ${ }^{3}$; José Iannacone ${ }^{4,5}$; Belkis E. Pérez Taboada ${ }^{1}$; Belkis García Cárdenas ${ }^{1}$; Máximo Santana Rodríguez ${ }^{1} \&$ Bárbara Pérez Arboláez ${ }^{1}$

1* Facultad de Tecnología de la Salud «Julio Trigo Lópę». Universidad de Ciencias Médicas «Dr. Serafin Ruiz. de Zárate Rui »» de Villa Clara, Cuba. rigobertofd@infomed.sld.cu

2 Centro Meteorológico Provincial de Villa Clara,Cuba. ricardo.oses@vcl.insmet.cu

3 Facultad de Ciencias Agropecuarias. Universidad Central «Marta Abreu» de Las Villas, Cuba. omelioc@uclv.edu.cu

$4 \quad$ Laboratorio de Parasitología. Facultad de Ciencias Biológicas. Universidad Ricardo Palma (URP). Lima, Perú.

5 Laboratorio de Ecología y Biodiversidad Animal. Universidad Nacional Federico Villarreal (UNFV). Facultad de Ciencias Naturalesy Matemática._joseiannacone@gmail.com

\section{ABSTRACT}

Leptospirosis constitutes a bacterial zoonosis of world distribution caused by a spirochete of the genus Leptospira that provokes frequent health problems humans and animals. Therefore, this research was conducted to determine the possible relationship between leptospirosis and some climatic variables in the municipality of Santa Clara, Villa Clara province from 2001 to 2014. The study was a descriptive observational transverse analysis of all of the epidemiological surveys applied to patients diagnosed with the illness being analyzed, characterizing the main sociodemographic and epidemiological variables. The Objective Regressive Regression (ROR) was used for the model. It was concluded that the presence of rodents in the home was the main infection source and the ages of highest risk between 15 to 59 years, fever, migraines, and arthromyalgias were the more frequently observed symptoms. Significant correlations were found between illness and the climatic variables in this study.

Key work: Epidemiological surveys - leptospirosis - Objective Regressive Regression (ROR) - Santa Clara - climatic variables

\section{RESUMEN}

La Leptospirosis constituye una zoonosis bacteriana de distribución mundial causada por una espiroqueta del género Leptospira, la cual provoca problemas de salud frecuentes en el hombre y los animales. Por ello se realiza esta investigación, con el objetivo de determinar la posible relación entre la entidad leptospirosis con algunas variables climáticas en el municipio Santa Clara, provincia Villa Clara durante el período comprendido desde el año 2001 al 2014. Para llevar a cabo dicho estudio se realizó una 
investigación observacional descriptiva transversal analizándose el total de las encuestas epidemiológicas aplicada a pacientes diagnosticados con la enfermedad, llegando a realizar la caracterización de las principales variables sociodemográficas y epidemiológicas. Se utilizó la Regresión Objetiva Regresiva (ROR) para la modelación. Se concluye que la presencia de roedores en el hogar fue la principal fuente de infección y las edades de mayor riesgo entre 15 a 59 años, la fiebre, cefaleas y artromialgias fueron los síntomas más frecuentes observados. Se encontraron correlaciones significativas entre la enfermedad con las variables climáticas en estudio.

Palabras clave: Encuestas epidemiológicas - leptospirosis - Regresión Objetiva Regresiva (ROR) - Santa Clara - variables climáticas

\section{INTRODUCCIÓN}

La distribución geográfica y la variación estacional de muchas enfermedades infecciosas son la primera evidencia de su relación con el tiempo y el clima (Rose, 1999). Las fluctuaciones y la variabilidad interanual del clima determinan el comportamiento de muchas de estas enfermedades consideradas por ello sensibles al clima (Rose, 1999; WHO, 2004).

Algunos estudios han mostrado que factores tales como la temperatura, las precipitaciones y la humedad originan cambios ecológicos que afectan directa o indirectamente el desarrollo de agentes patógenos, vectores y ciclos evolutivos que determinan finalmente la frecuencia, duración e intensidad de los focos infecciosos (Chandy et al., 2008); García et al., 2012.

Paralelamente, el propio hombre crea condiciones peligrosas a la salud al establecer escenarios sanitarios desfavorables, incorrectos asentamientos o erróneos patrones migratorios, alta densidad de población, contextos socioeconómicos deficientes y servicios de salud carentes de un enfoque preventivo. Algunos trabajos sólo muestran la asociación de las variaciones del clima y las enfermedades; pero, generalmente no contemplan la compleja dinámica causal de estos fenómenos para las predicciones y futuros escenarios de ahí que los sistemas de alerta temprana desarrollados no incluyen factores no climáticos e incluso de adaptación humana (Peñate, 2007; Osés et al., 2010).

La leptospirosis es una zoonosis, enfermedad bacteriana que afecta a los humanos y los animales. Es causada por la bacteria el género Leptospira de distribución mundial tanto en áreas urbanas como rurales (CONAVE, 2012; Joya et al., 2015; Haake \& Levett, 2015). Se estima globalmente que 10 mill se infectan de leptospirosis cada año y es difícil estimar exactamente cuántos de ellos mueren por este padecimiento, en gran medida por que los decesos ocurren en países donde las muertes no son sujetas a notificación rutinaria (Cintra et al., 2006; Rodríguez, 2011; Haake \& Levett, 2015). Esta es una enfermedad principalmente endémica en países con clima tropical y subtropical con amplio potencial epidémico. Se presenta frecuentemente con picos estacionales, algunas veces en brotes $y$ está asociada con cambios climáticos, principalmente inundaciones, inadecuadas condiciones de higiene y la ocupación o actividades recreativas (CONAVE, 2012; Joya et al., 2015; Haake \& Levett, 2015) y continúa siendo la de más amplia distribución mundial y la que mayores pérdidas ocasiona a la salud del hombre, a los animales y a la economía de los países (Martínez et al., 2004; Haake \& Levett, 2015). Su prevalencia real se desconoce, pero la infección se describe en más de 160 especies de mamíferos salvajes y domésticos (CaO et al., 2004; Adler et al., 2010).

La leptospirosis tiene una distribución mundial en los humanos, es endémica en muchos países tropicales y subtropicales y en los últimos 20 años se ha convertido en un verdadero problema de salud (Suputtamongkol et al., 2010; Haake \& Levett, 2015), presentándose por lo general, en forma de casos aislados o de brotes epidémicos (Chin, 2005; Adler et al., 2010).

En Cuba la leptospirosis humana tiene un comportamiento endemo-epidémico por ubicarse en el área tropical. Existen factores, que han favorecido la propagación de la leptospirosis en el hombre y los animales, tales como: el clima, el relieve, los diferentes fluviales naturales y artificiales, las extensas áreas agrícolas, los regímenes lluviosos en determinadas épocas del año, la crianza de animales domésticos, el fomento del perfil agropecuario, el número de trabajadores incorporados de forma temporal y permanente a estas tareas, la deficiente higiene ambiental imperante dada la 
inestabilidad en la recogida de los desechos sólidos y los cambios climáticos que afectan a la isla como parte del ecosistema (Céspedes, 2005).

En los últimos años, la leptospirosis ha afectado a un número considerable de personas de diferentes edades en el municipio Santa Clara, presentándose el mayor número de casos en el periodo lluvioso, que abarca desde el mes de mayo hasta el mes de octubre (CastilloCuenca et al., 2016).

No se dispone de una actualización a la caracterización del comportamiento de la leptospirosis en el municipio de Santa Clara en el período comprendido entre enero de 2001 hasta diciembre de 2014, considerando que la situación higiénico sanitaria deficiente favorece la aparición de la enfermedad en algunos consejos populares del municipio. Añadiendo que han ocurrido intensas lluvias e inundaciones debido al cambio climático. Se identifica la necesidad de incrementar los conocimientos sobre la leptospirosis para poder identificar los aspectos donde más impactos se puedan lograr para disminuir la morbilidad y mortalidad por la enfermedad en el territorio estudiado.

El objetivo de la investigación consistió en determinar la posible relación entre la entidad leptospirosis con algunas variables climáticas en el municipio Santa Clara, provincia Villa Clara, durante el período comprendido, desde el año 2001 al 2014.

\section{MATERIAL Y MÉTODOS}

Se realizó una investigación observacional descriptiva transversal con el objetivo de caracterizar epidemiológicamente la leptospirosis, así como la posible relación de esta entidad con algunas variables climáticas en el municipio Santa Clara, provincia Villa Clara, Cuba. El estudio abarcó el período comprendido desde el año 2001 al 2014. El universo estuvo constituido por el $100 \%$ de los pacientes notificados como confirmados de leptospirosis en dicho municipio.

Procedimientos para la recolección de la información

La recogida de la información se realizó a partir de la revisión documental al total de las encuestas epidemiológicas que le fueron aplicadas a cada paciente, que fueron notificados como leptospirosis confirmada. Esta encuesta está validada por expertos a nivel nacional y se aplica como parte del Programa Nacional de Control de la leptospirosis en el país.

\section{Condiciones de las viviendas}

- De mampostería y piso de cemento o mosaico

- Conexión al alcantarillado

- Agua tratada del acueducto

Buena: Cumple los tres requisitos.

Regular: Cumple dos requisitos.

Mala: Cumple con menos de dos requisitos.

Zona urbana: La ubicada dentro del perímetro urbano, en general de construcción continúa por la trama urbana y que puede contener áreas de uso comunal relativamente grande.

Zona Rural: Población reducida con densidad poblacional baja y casi siempre dispersa, con natalidad y mortalidad habitualmente más elevada que las zonas urbanas.

La técnica serológica empleada fue la Hemoaglutinación Pasiva (HA), la cual se le aplica a los pacientes con cuadro clínico epidemiológico de leptospirosis, para la misma se toma una primera muestra y a los 14-15 días se toma la segunda, siendo el resultado positivo si se cuadriplican los títulos de anticuerpo contra la Leptospira, dicha técnica tiene como inconvenientes las acciones cruzadas con otros microorganismos (Peñate, 2007).

Se tuvo en cuenta para el caso de la posible relación de la entidad leptospirosis con algunas variables climáticas, el registro de datos existentes en la estación meteorológica ubicada en el área del valle del Yabú, en la ciudad de Santa Clara, Cuba.

\section{Procesamiento de la información}

La información obtenida del análisis de estas encuesta fue procesada mediante el programa MS Excel 2010 y se incluye en una base de datos del programa SPSS para el SO Windows, V 13.0.

Se emplearon las técnicas de la estadística descriptiva para valorar la caracterización de las diferentes variables sociodemográficas y clínico-epidemiológicas confeccionándose tablas y gráficos donde se representaran los resultados expresados en frecuencia absoluta (valor) en frecuencia relativa (por ciento). 
Para asociar la morbilidad por leptospirosis con las diferentes variables climatológicas, las variables utilizadas: temperaturas (Media, Máxima y Mínima), humedades (Media, Máxima y Mínima), la precipitación total en todo el periodo a tratar y la radiación global, que se obtuvo en el periodo comprendido de 2005-2010, tomada de la estación de Camagüey, que es la única que presenta datos relativos aplicables a nuestra provincia.

Primeramente, se utilizó la metodología ROR (Osés \& Grau, 2011) para la modelación de la variable leptospirosis total en el año, posteriormente se calcularon las correlaciones de los errores con las variables climáticas para ver cuál de ellas era la más significativa, se analizó la cross-correlación de las variables climáticas con los errores del modelo para analizar el impacto de las variables climáticas, debido a la poca cantidad de casos no se utilizó una muestra independiente, para la modelación se utilizó el paquete estadístico SPSS 13. Se realizó un pronóstico para el año 2015 y 2016.

Se analizó la incidencia de dichas variables sobre la entidad objeto de estudio. La información de las variables climáticas se obtuvo en el Instituto de Meteorología Provincial de Villa Clara, perteneciente a la Academia de Ciencias de Cuba.

\section{RESULTADOS Y DISCUSIÓN}

Se analizaron un total de 91 pacientes afectados por Leptospira en el periodo enmarcado de 2001 al 2014 (Tabla 1), donde se reflejan los datos del grupo de pacientes según los atributos de edad y sexo. Se aprecia superioridad para rangos de edades comprendidos entre los 15 y 59 años (63,74\%), considerando que estas edades están estrechamente vinculadas a actividades de mayor riesgo para la adquisición de la enfermedad. Entre estas actividades están: labores agrícolas, actividades recreativas, pesca en ríos, presas u otros tipos de embalses, dicho resultado coincide con lo planteado por Bollet et al. (2003), quienes aseguran, que los hombres en edades jóvenes resultan ser los más afectados.

Por su parte Dos Santos et al. (2003) afirman que las personas de mayor riesgo a enfermar son las que tienen edades comprendidas entre 18 y 60 años. No obstante, Suárez (1999) plantea que existe un alto sub-registro, tanto en niños como adultos, ya que el pleomorfismo clínico de la enfermedad es muy variado y la exposición a posibles fuentes de infección es amplia. Además según estudios de seroprevalencia se ha encontrado que un número elevado de infantes de segunda y tercera infancia presentan anticuerpos antileptospira, lo que demuestra que han tenido en algún momento contacto con el germen y posiblemente la infección esté más difundida de lo que se crea.

Tabla 1. Relación entre grupos de edades y sexo.

\begin{tabular}{|c|c|c|c|c|c|c|c|c|}
\hline \multirow{3}{*}{ Sexo } & \multicolumn{8}{|c|}{ Grupo de edades } \\
\hline & \multicolumn{2}{|c|}{ 0-14 Años } & \multicolumn{2}{|c|}{ 15-59 Años } & \multicolumn{2}{|c|}{60 y más } & \multicolumn{2}{|c|}{ Total } \\
\hline & $\#$ & $\%$ & $\#$ & $\%$ & \# & $\%$ & $\#$ & $\%$ \\
\hline Masculino & 16 & 100 & 40 & 68,97 & 11 & 64,71 & 67 & 73,63 \\
\hline Femenino & 0 & 0 & 18 & 31,03 & 6 & 35,29 & 24 & 26,37 \\
\hline Total & 16 & 17,58 & 58 & 63,74 & 17 & 18,68 & 91 & 100 \\
\hline
\end{tabular}

Fuente: Unidad Municipal de Higiene y Epidemiología de Santa Clara.

El mayor número de casos correspondió al sexo masculino con un $73,63 \%$ del total de casos confirmados, siendo el sexo opuesto menos afectado por la enfermedad. En relación con el grupo etario de mayor frecuencia de presentación de la leptospirosis, otros investigadores en Cuba también apreciaron superioridad en el intervalo comprendido entre 15 a 59 años (Chamizo et al., 1996).
La elevada frecuencia de presentación de la leptospirosis en el sexo masculino está relacionada con las actividades citadas anteriormente, aspecto que también ha sido revelado en estudios realizados en Costa Rica (Valverde et al., 2008) y en Cuba (Padilla et al., 2003, Martínez et al., 2004). En estos trabajos se afirma que el sexo masculino presenta mayor riesgo de contraer la enfermedad, por ser el más expuesto a las distintas fuentes de infección. 
En la muestra analizada se aprecian las diferentes áreas de salud del municipio (Tabla 2), donde se identifica, que el mayor número de enfermos se encuentra ubicado en el área de salud "Capitán Roberto Fleites", con un total de 26 casos notificados como confirmados de la enfermedad, para un $28,57 \%$. De estos, un $17,58 \%$ son del sexo masculino y el $10,99 \%$ del sexo opuesto. Esto se debe a que esta área de salud abarca un mayor universo, tanto rural como urbano, y por ello existe un número mayor de habitantes totales.

Tabla 2. Distribución de la leptospirosis según áreas de salud y sexo.

\begin{tabular}{|c|c|c|c|c|c|c|}
\hline \multirow{3}{*}{ Áreas de Salud } & \multicolumn{5}{|c|}{ Sexo } & \\
\hline & Masculino & & men & & & \\
\hline & $\mathrm{N}$ & $\%$ & $\mathrm{~N}$ & $\%$ & $\mathrm{~N}$ & $\%$ \\
\hline Polic. Roberto Fleites & 16 & 17,58 & 10 & 10,99 & 26 & 28,57 \\
\hline Polic. XX Aniversario & 12 & 13,19 & 4 & 4,40 & 16 & 17,58 \\
\hline Polic. Marta Abreu & 16 & 17,58 & 0 & 0 & 16 & 17,58 \\
\hline Polic. Chiqui Gómez & 13 & 14,29 & 2 & 2,20 & 15 & 16,48 \\
\hline Polic. José R. León Acosta & 7 & 7,69 & 4 & 4,40 & 11 & 12,09 \\
\hline Polic. Santa Clara & 3 & 3,30 & 4 & 4,40 & 7 & 7,69 \\
\hline Total & 67 & 73,63 & 24 & 26,37 & 91 & 100 \\
\hline
\end{tabular}

Leyenda: Polic. Policlínico.

Fuente: Unidad Municipal de Higiene y Epidemiología de Santa Clara.

En un estudio clínico-epidemiológico realizado por Leptospirosis Working Group (2002) en la comunidad de Springfield, Illinois, EUA, se reveló que en un brote de la enfermedad entre participantes de un triatlón y residentes de la comunidad, la mayoría de los pacientes manifestó haber tenido contacto con roedores o convivencia con éstos. En investigaciones realizadas en Barbados (Levett, 1999) y en Panamá (Vanasco et al., 2007), coinciden al afirmar, que la enfermedad tiene una alta prevalencia donde existe un alto índice de roedores peri-domiciliarios, aunque otros animales como: perros, cerdos y bovinos son capaces de excretar leptospiras por la orina y heces fecales, tanto durante la enfermedad activa como durante el período de portador asintomático.

El estudio realizado en cuanto al análisis de los factores de riesgo asociados a los casos confirmados de leptospirosis reveló, que el contacto con roedores sinantrópicos en las viviendas alcanzó el 36,26\%, seguido del baño y pesca en ríos o presas 32,97\% (figura 1). Este resultado coincide con Martín et al (2002) en un estudio clínico epidemiológico, donde la mayoría de los pacientes manifestó haber tenido contacto con roedores y/o convivencia con ellos y donde, la principal fuente de infección para el hombre fueron las ratas, roedores silvestres, los perros, cerdos y bovinos. Estos animales excretan la Leptospira por la orina y las heces fecales, tanto durante la enfermedad activa como en el periodo de portador asintomático. Además, Levett et al (2003) plantean que la enfermedad tiene una alta prevalencia donde existe un alto índice de roedores peri-domiciliarios.

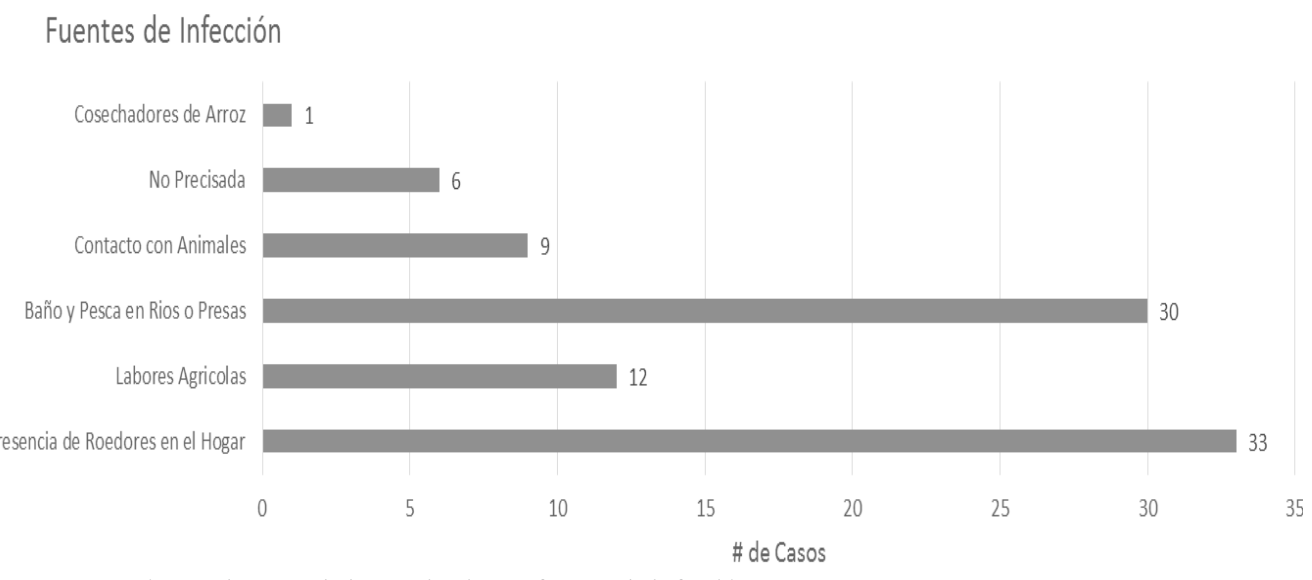

Figura 1. Número de casos de leptospirosis por fuentes de infección. 
En la figura 2 se aprecian los síntomas más frecuentes presentes en el total de casos ocurridos durante los 14 años de estudio, estos corresponden a la fiebre $(97,80 \%)$, cefalea $(73,63 \%)$ y mialgia $(67,03 \%)$. Los síntomas más frecuentes en el total de los casos ocurridos en el decenio fueron: fiebre, cefalea y mialgia, lo cual coincide con los resultados obtenidos por Speelman (2000), Zunino et al. (2007) \& Peñate (2007) en sus trabajos sobre leptospirosis. Los restantes síntomas se presentaron en menor porcentaje.

\section{Sintomatología Clínica}

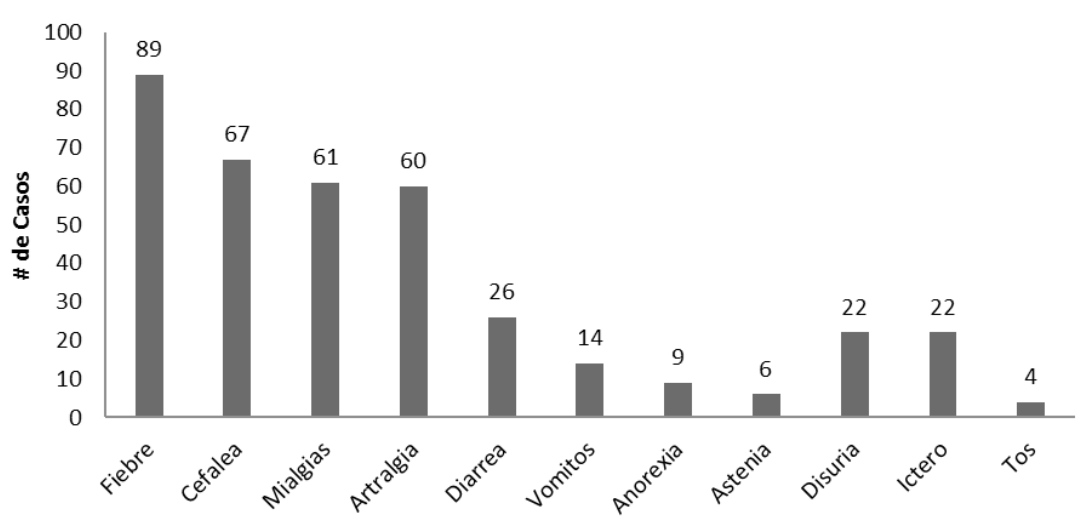

Figura 2. Distribución de los pacientes según sintomatología clínica.

En cuanto a la distribución de casos de leptospirosis por años y meses, se identificó que fueron los años 2003 y 2008 los de mayor morbilidad (figura 3); además, se observa que los meses de junio, julio, septiembre y octubre fueron los de mayor incidencia en cuanto al número de casos, lo que coincide con el periodo lluvioso en Cuba (figura 4). Estos resultados coinciden con los obtenidos por Valverde et al. (2008), quienes plantean, que la leptospirosis es más frecuente durante la estación lluviosa favorecida por un incremento en la exposición con aguas estancadas. Los meses de menor ocurrencia de casos estuvieron enmarcados en el periodo poco lluvioso y el mes de agosto.

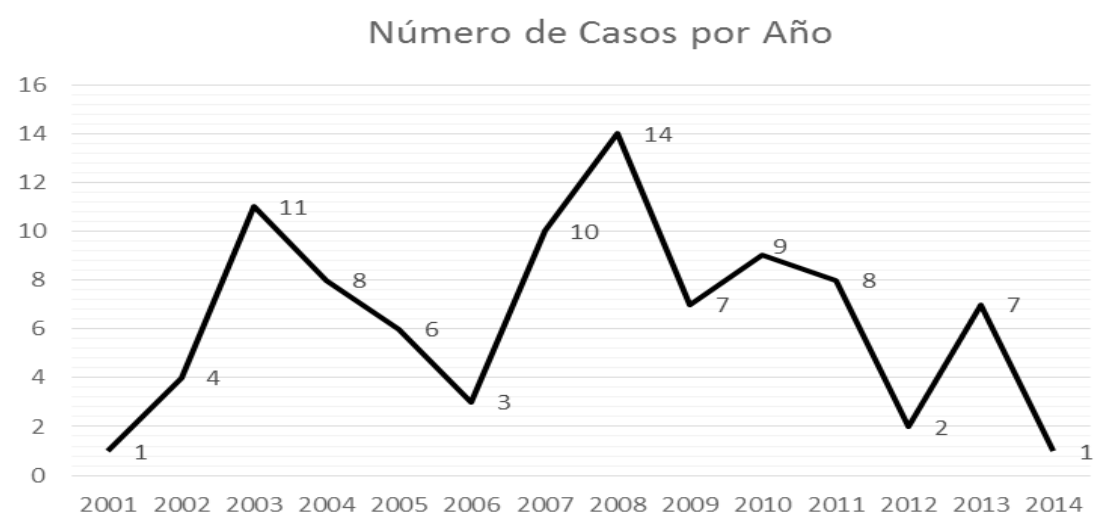

Figura 3. Casos de Leptospirosis por año. 


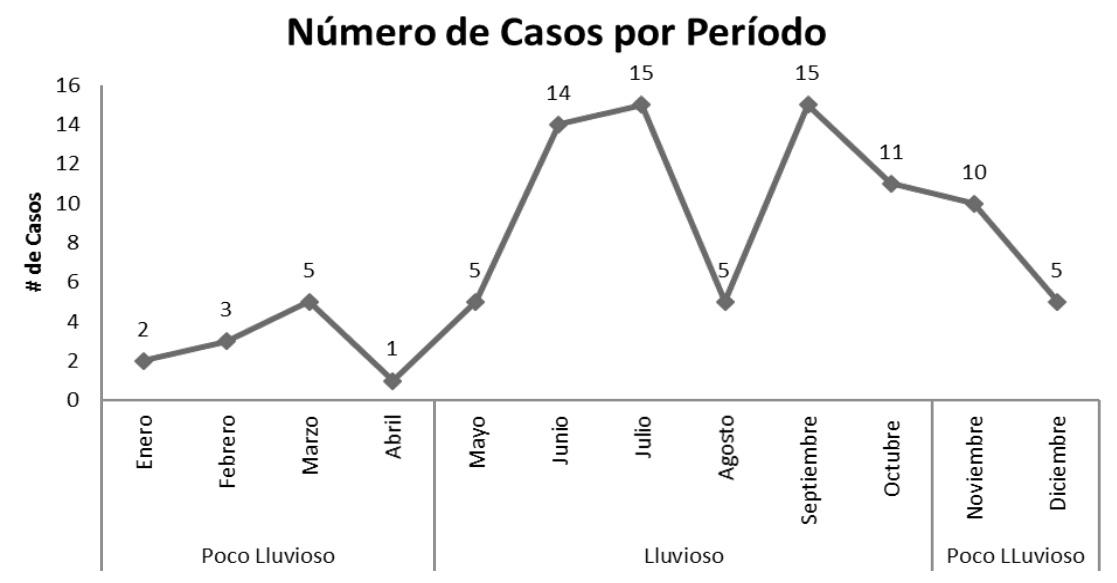

Figura 4. Casos de Leptospirosis por periodos y meses del año.

Las condiciones de vivienda de los enfermos en el periodo analizado fueron: buenas $(64,84 \%)$, regular $(26,37 \%)$ y malas $(8,79 \%)$. En nuestro estudio sobre condiciones de la vivienda, no coincidimos con los resultados de Martín et al (2002), el cual asegura que las personas que presentaban viviendas con estructura precaria eran los más propensos de enfermar. Sin embargo, coincidimos con lo planteado por Speelman (2000), en relación a las personas que viven en zonas periféricas de ciudades con malas condiciones higiénicas, que son las que tienen mayor riesgo de enfermar. Acorde los resultados obtenidos en el presente trabajo, no son las condiciones precarias de la vivienda las que inciden en la presentación de la enfermedad, sino la exposición ocupacional a las diferentes fuentes de infección.

El estudio llevado a cabo en este trabajo permite señalar que los consejos populares con mayor cantidad de casos reportados fueron: Condado Sur y CamachoLibertad, ubicado en la zona urbana con el 9,98\% de casos confirmados y el consejo popular Hatillo Yabú, ubicado en la zona rural del municipio, con un 6,59\% de casos reportados. Con un total de 73 pacientes en la zona urbana y 18 casos de la zona rural en total, dichos resultados contrastan con los obtenidos por Morgan (2002), quien plantea que la enfermedad se presenta mayoritariamente en las zonas rurales.
Relacionado con la ocupación, se obtiene como resultado, que los estudiantes son los más afectados, con un $26,37 \%$ del total seguido por otras labores como: personal veterinario, militar, trabajadores de alcantarillado y de matadero, lo cual representaron el $21,98 \%$ y los obreros agrícolas 16,48 \% (figura 5).

Concordamos con Morgan (2002) en cuanto a lo relacionado con las actividades ocupacionales, donde considera el mayor riesgo de contraer la enfermedad en aquellos grupos expuestos, como trabajadores de arrozales, cañaverales, alcantarillados, médicos veterinarios, personal militar y otros. Suárez (1999) y Bender \& Hall (2003) aseguran, que en el caso particular de los estudiantes pueden infectarse en actividades recreativas al entrar en contacto con agua dulce estancada contaminada (baño, pesca y deportes acuáticos) y por contacto con mascotas. Levett (2003) describe un brote en Illinois de una enfermedad febril aguda en 44 personas, donde el agente causal fue la Leptospira, enfermando así, atletas y personas con la exposición ocupacional y recreativa en el lago Springfield, donde tuvo lugar el suceso. Difiriendo de Tappero et al. (2000), el cual plantea, que las personas que se dedican a trabajos agrícolas en las zonas rurales son las que tienen mayor riesgo de enfermar. 


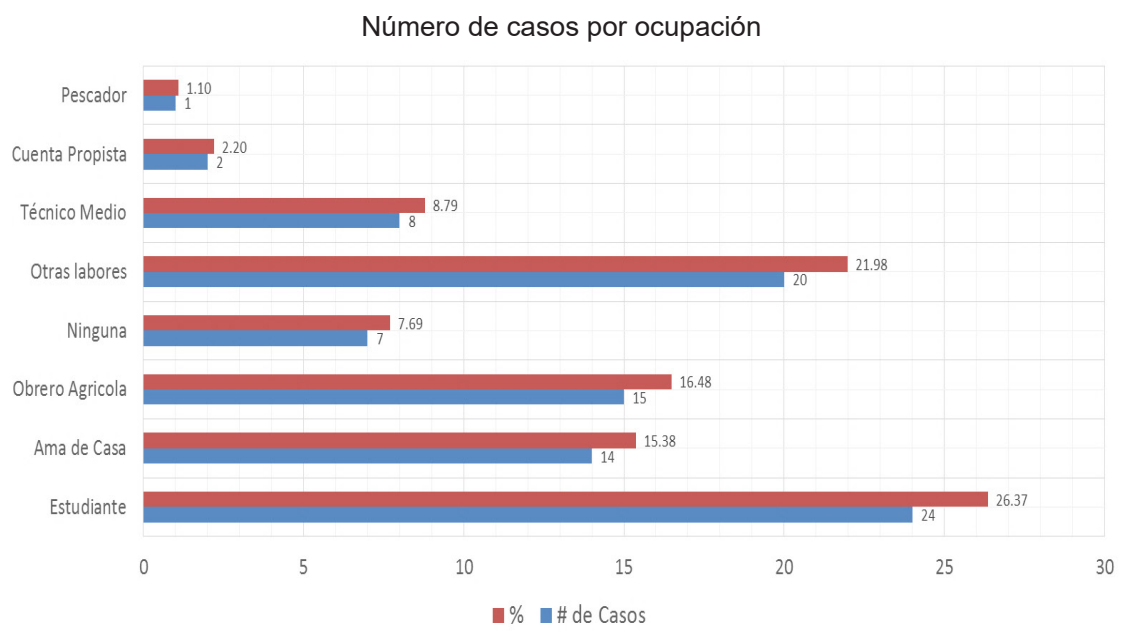

Figura 5. Casos de leptospirosis por ocupación laboral.

En el presente trabajo se utiliza como modelo la regresión objetiva regresiva, dando como resultado, que la varianza explicada $100 \%$ con un error estándar de cero casos, donde el estadístico de Durbin Watson es cercano a 2, por lo que no existe correlación entre los errores (tabla 3 ).

Tabla 3. Algunos estadísticos del modelo estadístico.

Resumen del Modelo ${ }^{\mathrm{c}, \mathrm{d}}$

\begin{tabular}{cccccc}
\hline Modelo & $\mathrm{R}$ & R Cuadrado $^{\mathrm{a}}$ & $\begin{array}{c}\text { R Cuadrado } \\
\text { Ajustada }\end{array}$ & $\begin{array}{c}\text { Error Estándar del } \\
\text { Estimado }\end{array}$ & $\begin{array}{c}\text { Durbin- } \\
\text { Watson }\end{array}$ \\
\hline 1 & $1,00^{\mathrm{b}}$ & 1,000 & . &. & 1,47 \\
\hline
\end{tabular}

a. Regresión directo del origen, R Cuadrado medidas de la proporción de la variabilidad en la variable dependiente acerca del origen explicado por regresión.

b. Predictores: Lag4 Precipitación, DS, Lag2 Leptospirosis, NoC, Lag4 Radiación, DI

c. Variable Dependiente: Casos.

d. Regresión Linear directo del Origen.

El Análisis de Varianza fue significativo al $100 \%$, con una F de Fisher no determinada (tabla 4), para profundizar y comprender este tipo de modelo recomendamos Osés \& Grau (2011), donde se muestra una forma matemática de resolver cuando los errores son cero y se plantea una división por cero de los parámetros introduciendo los números Cristosoles (Números divididos por cero).

Tabla 4. Análisis de varianza del modelo.

ANOVA ${ }^{\mathrm{c}, \mathrm{d}}$

\begin{tabular}{|c|c|c|c|c|c|c|}
\hline Modelo & & $\begin{array}{c}\text { Suma de } \\
\text { Cuadrados }\end{array}$ & $\mathrm{gl}$ & Cuadrado Medio & $\mathrm{F}$ & Sig. \\
\hline \multirow[t]{3}{*}{1} & Regresión & 248,00 & 6 & 41,33 & . &.$^{a}$ \\
\hline & Residual & 0,00 & 0 & -3 & & \\
\hline & Total & $248,00^{\mathrm{b}}$ & 6 & & & \\
\hline
\end{tabular}

Predictores: Lag4 Precipitación, DS, Lag2 Leptospirosis, NoC, Lag4 Radiación, DI.

b. Esta suma total de cuadrados no está corregida por la constant porque la constante es cero para la regresión a través del origen.

c. Variable Dependiente: Casos.

d. Regresión Linear directo del Origen. 
El modelo expuesto en la tabla 5 donde la DS y DI explican los altibajos de la serie, siendo ambas significativas al $100 \%$, mientras NoC representa la tendencia en el tiempo la cual es significativa a la disminución, lag2Leptospirosis, significa la cantidad de casos de leptospirosis regresada en dos años, entraron también lag4Radiacion, que significa la radiación total regresada en cuatro años y lag4Precipitación, que es la precipitación total en el año, regresada en cuatro años; este modelo explica el $100 \%$ de los casos, pero debido a que no contamos con datos de radiación en el 2014 y 2015 para poder pronosticar, finalmente utilizamos un modelo alternativo para el pronóstico del 2015 y 2016. La leptospirosis regresada en dos años influye en el modelo final, así como la precipitación regresada en 4 años y la radiación total regresada en 4 años.

Tabla 5. Modelo para la leptospirosis en el municipio Santa Clara.

\begin{tabular}{|c|c|c|c|c|c|c|}
\hline & & $\begin{array}{l}\text { Coeficientes sin } \\
\text { Estand. }\end{array}$ & & $\begin{array}{c}\text { Coeficiente } \\
\text { Estandarizado }\end{array}$ & & \\
\hline Modelo & & $\mathrm{B}$ & $\begin{array}{c}\text { Error } \\
\text { Estándar }\end{array}$ & Beta & $\mathrm{t}$ & Sig. \\
\hline & DS & 162,14 & 0,00 & 17,83 & . & . \\
\hline & DI & 168,95 & 0,00 & 18,58 & . & . \\
\hline & $\mathrm{NoC}$ & $-5,76$ & 0,00 & $-10,42$ & . & . \\
\hline & Lag2 Leptospirosis & $-1,78$ & 0,00 & $-2,51$ & . & . \\
\hline & Lag4 Radiación & $-0,14$ & 0,00 & $-7,75$ & . & . \\
\hline & Lag4 Precipitación & $-0,02$ & 0,00 & $-4,54$ & . & . \\
\hline
\end{tabular}

Como puede verse, los residuales estandarizados en la tabla 6 presentan media cero y desviación estándar uno. Este modelo obtenido es ampliamente superior al obtenido por García et al. (2012) para este mismo municipio de Santa Clara, ya que el índice de varianza explicada es superior con menor error para el modelo.

Tabla 6. Residuales del modelo de leptospirosis.

Residuales Estadísticos ${ }^{\mathrm{a}, \mathrm{b}}$

\begin{tabular}{|c|c|c|c|c|c|}
\hline & Mínimum & Máximum & Media & $\begin{array}{c}\text { Desviación } \\
\text { Estándar }\end{array}$ & $\mathrm{N}$ \\
\hline Valor Predicho & 1,00 & 9,00 & 5,67 & 3,32 & 6 \\
\hline Residual & 0,00 & 0,00 & 0,00 & 0,00 & 6 \\
\hline $\begin{array}{l}\text { Std. Valor } \\
\text { Predicho }\end{array}$ & $-1,40$ & 1,00 & 0,00 & 1,00 & 6 \\
\hline Std. Residual & . & . & . & . & 0 \\
\hline
\end{tabular}

a. Variable Dependiente: Casos

b. Regresión Linear directo del Origen.

Posteriormente se calcularon las correlaciones con las variables climáticas, donde se aprecia que ninguna variable fue significativa, por lo que se hace necesario para posteriores trabajos, buscar si existe alguna variable climática que pueda ser significativa para usarla en el pronóstico, hasta aquí este resultado difiere con respecto a otros autores (Osés et al., 2010). En donde se emplean variables climáticas, quizás los resultados difieren debido a que en este último trabajo se emplearon datos mensuales que hacen que la estadística aumente, en nuestro trabajo los datos son anuales solamente, debido a que no contamos con los datos de radiación del 2014 y 2015. Se utilizó un modelo alternativo para predecir el comportamiento de la leptospirosis en el municipio (tabla 7). 
Tabla 7. Correlación de los residuales con las variables climáticas.

\begin{tabular}{lcc}
\hline \multicolumn{1}{c}{ Variables } & Correlación & Significación \\
\hline Temperatura Máxima & 0,08 & 0,76 \\
Temperatura Mínima & 0,23 & 0,42 \\
Temperatura Media & 0,03 & 0,91 \\
H.R. Máxima & $-0,23$ & 0,93 \\
H.R. Mínima & 0,13 & 0,65 \\
H.R. Media & 0,09 & 0,75 \\
Precipitación Anual & 0,23 & 0,42 \\
\hline
\end{tabular}

El modelo alternativo utiliza la Crosscorrelación con la variable precipitación total en el año; en la figura 6 se aprecia este resultado, donde el retardo 4 es el más significativo.

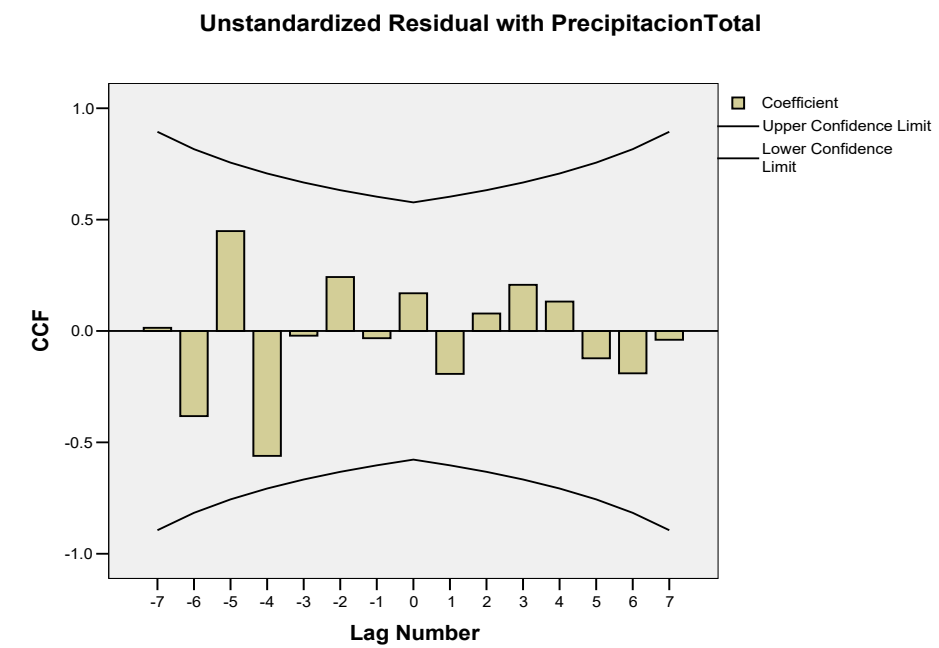

Figura 6. Crosscorrelación del residual con la precipitación total. Coefficient $=$ Coeficiente Upper Confidence Limit = Límite de confianza superior. Lower Confidence Limit = Límite de confianza inferior.

Por tanto, el modelo resultante explica el 0,90 de Varianza con un error de 4,68 casos, donde el análisis de varianza dio como resultado una $\mathrm{F}$ de Fisher de 4,358 significativa al $90 \%$ (tabla 8 ).

Tabla 8. Análisis de varianza del nuevo modelo.

ANOVAc,d

\begin{tabular}{llccccc}
\hline \multirow{2}{*}{ Modelo } & & $\begin{array}{c}\text { Suma de } \\
\text { Cuadrados }\end{array}$ & gl & $\begin{array}{c}\text { Cuadrado } \\
\text { Medio }\end{array}$ & F & Sig. \\
\hline \multirow{2}{*}{1} & Regresión & 479,06 & 5 & 95,81 & 4,35 & $0,06^{\mathrm{a}}$ \\
& Residual & 109,93 & 5 & 21,98 & & \\
& Total & $589,00^{\mathrm{b}}$ & 10 & & & \\
\hline
\end{tabular}

a. Predictores: Lag4 Precipitación, DI, Lag2 Leptospirosis, NoC, DS.

b. Suma total de cuadrados no es correcta para la constante, porque la constante es cero por regresión directa del origen.

c. Variable Dependiente: Casos.

d. Regresión Linear directo del Origen.

En la tabla 9 se reflejan los valores anuales de leptospirosis y los valores predichos, estos últimos siguen en gran medida los ascensos y descensos de la leptospirosis. Los valores predichos para el 2015 y 2016 se prevén con tendencia a la disminución del número de casos futuros. Predictivamente se puede comentar que para el 2015 se presentaran cinco casos notificados como confirmados y tres para el 2016. La tendencia de la leptospirosis será a la disminución en 5,7 casos en los próximos dos años. 
Tabla 9. Marcha anual de la leptospirosis y valor predicho.

Resumen de Casos ${ }^{a}$

\begin{tabular}{ccccc}
\hline & Años & Casos & $\begin{array}{c}\text { Valor Predicho sin } \\
\text { Estandarizar }\end{array}$ & $\begin{array}{c}\text { Residual sin } \\
\text { Estandarizar }\end{array}$ \\
\hline 1 & 2001 & 1 & $\cdot$ & $\cdot$ \\
2 & 2002 & 4 & $\cdot$ & $\cdot$ \\
3 & 2003 & 11 & $\cdot$ & $\cdot$ \\
4 & 2004 & 8 &. &. \\
5 & 2005 & 6 & 8,92 & $-2,92$ \\
6 & 2006 & 3 & 8,21 & $-5,21$ \\
7 & 2007 & 10 & 7,59 & 2,40 \\
8 & 2008 & 14 & 8,22 & 5,77 \\
9 & 2009 & 7 & 6,96 & .036 \\
10 & 2010 & 9 & 4,47 & 4,52 \\
11 & 2011 & 8 & 7,05 & .94 \\
12 & 2012 & 2 & 4,05 & $-2,05$ \\
13 & 2013 & 7 & 7,45 & -.45 \\
14 & 2014 & 1 & 4,04 & $-3,04$ \\
15 & 2015 & $\cdot$ & 5,36 & $\cdot$ \\
16 & $\cdot$ &. & 3,57 &. \\
\hline Total (N) & 15 & 14 & 12 & 10 \\
\hline
\end{tabular}

a. Limitado para los primeros 100 casos.

\section{REFERENCIAS BIBLIOGRÁFICAS}

Adler, B. \& De la Peña, A. 2010. Leptospira and leptospirosis. Veterinary Microbiology, 140: 287-296.

Bender, L. \& Hall, P. 2003. Leptospira interrogans exposure in free ranging skin. Washington. Journal of Wildlife Diseases, 32: 121-124.

Bollet, A.; González, R. \& Pérez, A. 2003. Leptospirosis humana, Un problema de Salud. Revista Biomédica, 15: 140-146.

Cao, P.; Parellada, B.; Padrón, S.; Véliz, P.; Guzmán, M. \& Jorna, A. 2004. Comportamiento de la leptospirosis grave en la unidad de cuidados intensivos. Revista Cubana de Medicina, 43: http://www.scielo.sld.cu/scielo.php. Consultado el: 4/12/2014.

Castillo-Cuenca, J.C; Iannacone, J.; Fimia-Duarte, R.; Quiñones-Prieto, M.C; Cepero-Rodríguez, O.; Cruz-Rodríguez, D. \& Campos-Cardoso, L.M. 2016. Epidemiological behavior of human and animal leptospirosis in Villa Clara, Cuba. The Biologist (Lima), 14: 89-102.
Céspedes, M. 2005. Leptospirosis: enfermedad zoonótica emergente. Revista Peruana de Medicina Experimental y Salud Publica, 22: 290-307.

Chamizo, G.H.; Cruz, P.R. \& Borroto, P.R. 1996. Estudio geoepidemiológico de la leptospirosis humana en Cuba. Revista Cubana de Higiene y Epidemiología, 34: 15-22.

Chandy, S.; Boorugu, H.; Chrispal, A.; Thomas, K.; Abraham, P. \& Sridharan, G. 2009. Hantavirus infection: a case report from India. Indian Journal of Medical Microbiology, 27: 267270.

Chin, J. 2005. El control de las enfermedades transmisibles. OPS, Washington, DC.

Cintra, M.; Pérez, L.; Suárez, Y.; Soca, M. \& Martínez, A. 2006. La zoonosis como ciencia y su impacto social. Revista Electrónica de Veterinaria, REDVET, 7: http://www.veterinaria.org/ revistas/redvet/n090906.html 
CONAVE (Comité Nacional para la Vigilancia Epidemiológica). 2012. Manual de Procedimientos Estandarizados para la Vigilancia Epidemiológica de la Leptospirosis. Dirección General de Epidemiología. E.U.M.

Dos Santos, M.V.; Dos Santos, J.A.; Sugai, T.A. \& Dos Santos, A.L. 2003. Síndrome de Weil. Revista Cubana de Medicina Tropical, 55: 44-46.

Haake, D.A. \& Levett, P. N. 2015. Leptospirosis in Humans. Current Topics in Microbiology and Immunology, 387: 65-97.

Joya, L.C.; Gutierrez, D.L.; Hurtado, W.M. \& Gama, J.M. 2015. Leptospira: revisión del agente causal de una enfermedad zoonótica. Biociencias, 10: $65-80$.

Levett, P. 1999. Leptospirosis: re-emerging or rediscovered disease?. Journal of Medical Microbiology, 48: 417-418.

Levett, P.; Walton, D. \& Waterman , L. 2003. Surveillance of leptospiral carriage by feral rats in Barbados. West Indian Medical Journal, 41: 15-17.

Martín, U.; Sansevy, A.; Colombo, J. \& Romantin, V. 2002. Leptospirosis en la provincia de Santa Fe. Descripción epidemiológica, clínica y socioeconómica. Medicina, 62: 164-168.

Martínez, R.; Pérez, A.; Quiñones, M.; Cruz, R.; Álvarez, A.; Armesto, M.; Fernández, C.; Menéndez, J.; Rodríguez, I.; Baró, M.; Díaz, M.; Rodríguez J.; Sierra, G.; Obregón, A.M.; Toledo, M.E. \& Fernández, N. 2004. Efficacy and safety of a vaccine against human leptospirosis in Cuba. Revista Panamericana de Salud Pública, 15: 249-255.

Morgan, J. 2002. Leptospirosis en comunidades rurales. Clinical Infectious Diseases, 34: 1120-1129.

Osés, R.; Bonet, J.; Cepero, O.; Saura, G. \& Pedraza, A. 2010. Evaluación del comportamiento de la leptospirosis humana mediante un modelo matemático atendiendo a variables climáticas como predictoras. Revista Electrónica de Veterinaria, REDVET, 11: 1-12.
Oses, R. \& Grau, R. 2011. Modelación regresiva (ROR), versus modelación ARIMA, usando variables dicotómicas en mutaciones del VIH. Universidad Central Marta Abreu de las Villas.

Padilla, P.; Vila, C.; Vidal, G. \& Rodríguez, A. 2003. Leptospirosis, mortalidad en Cuba. Revista Cubana de Medicina Tropical, 50: 61-65.

Peñate, M. 2007. Leptospirosis bumana. En: Diagnóstico y tratamiento. Medicina Interna. $1^{\mathrm{a}}$ ed. La Habana: Editorial Ciencias Médicas, 534-37.pp.

Rodríguez, A.P. 2011. Variabilidad y cambios climáticos. Impacto sobre algunas enfermedades infecciosas Habana Ciencias Medicas, 10: 372-381

Rose, J. 1999. Health, climate and infectious disease; a global perspective. A report from American Academy of Microbiology, Tucson, Arizona.

García, G.S.; Pérez, B.J.; Fimia, D.R.; Osés, R.R.; Garín, L.G. \& González, G.R. 2012. Influencia de algunas variables climatológicas sobre las densidades larvarias en criaderos de culícidos. Pol. Cap. Roberto Fleites. Santa Clara 20092010. Revista Electrónica de Veterinaria, REDVET, 13 (05B). Disponible en: http:// www.veterinaria.org/revistas/redvet

Speelman, P. 2000. Leptospirosis. En: Harrison Principios de medicina interna. $14^{\text {ta }}$ ed. Madrid: McGrawHill, 1187-1189.

Suárez, M. 1999. Estudio de leptospirosis en niños de Ciego de Ávila. Revista Clínica Española, 194: 433-439.

Suputtamongkol, Y.; Pongtavornpinyo, W.; Lubell, Y.; Suttinont, C.; Hoontrahul, S.; Phimda, L.; Losuwanaluk, K.; Suwancharoen, D.; Silpasakorn, S.; Chierakul, W. \& Day, N. 2010. Strategies for diagnosis and treatment of suspected leptospirosis: a cost-benefit analysis. PLOS Neglected Tropical Diseases, 4: 1-6.

Tappero, J.; Ashford, D. \& Perkins, B. 2000. Leptospira species. Douglas and Bennett s principles and practice of infections Diseaces. 2: 2495-2502. 
Valverde, M.; Brenes, R. \& Sequeiro, J. 2008. Confirmación por laboratorio de leptospirosis. Acta Médica Costarricense, 50: 51-54.

Vanasco, N.; Lottersberger, J.; Schmelinmf, A., Gardner, I. \& Tarabla, H. 2007. Diagnóstico de la Leptospirosis: Evaluación de un enzimo inmunoenzayo en fase sólida en diferentes etapas de la enfermedad. Revista Panamericana de Salud Pública, 21: 388- 395.
WHO 2004. Using climate to Predict Infectious Disease Outbreaks: A Review. Geneva, WHO/SDE/ $\mathrm{OEH} / 04.01$.

Zunino, E. \& Pizarro, R. 2007. Leptospirosis: puesta al día. Revista chilena de infectologia, 24: 220226.

Received October 8, 2016.

Accepted December 13, 2016. 